\title{
Field testing of the use of INTAKE24 - an online 24 hour dietary recall system
}

\author{
M.K. Rowland ${ }^{1}$, E. Simpson ${ }^{1}$, A.J. Adamson ${ }^{1}$, I. Poliakov ${ }^{2}$, P. Olivier ${ }^{2}$ and E. Foster ${ }^{1}$ \\ ${ }^{1}$ Human Nutrition Research Centre, Institute of Health \& Society, Newcastle University, Newcastle upon Tyne NE2 \\ 4HH, UK and ${ }^{2}$ Open Lab, School of Computing Science, Newcastle University, Newcastle upon Tyne NE1 8HW, UK
}

INTAKE24 is an online 24-hour dietary recall system which allows users to self-report their dietary intake. The system has undergone relative validation against interview led 24-hour recalls in 167 11-24 year olds, where good agreement between the two methods was found $^{(1)}$. In order to develop the system for use with the general adult population and to improve the usability of the system in all age groups, a field test of INTAKE24 in those aged from 11 years up to and including older adults, was conducted. The field test assessed the suitability of INTAKE24 as a system for collecting dietary information from a diverse population and to collect extensive user feedback to inform further development. Participants were asked to complete two week days and two weekend days of INTAKE24 on non-consecutive days over a 10-day period.

Of those who agreed to take part in the study, $\sim 60 \%(\mathrm{n}=230)$ completed at least one recall, and $\sim 50 \%(\mathrm{n}=195)$ completed two or more recalls. Nutrient intakes for adults and children were similar to those found in other dietary surveys (e.g. the Low Income Diet and Nutrition Survey ${ }^{(2)}$ ) with the $\%$ NMES and fat as a $\%$ energy within $3 \%$ of those found in LIDNS. The $\%$ energy intake for males was within $15 \%$ and for females it was within $2 \%$.

Answers from the feedback questionnaire $(n=185)$ showed that the majority of users agreed or strongly agreed that they would like to use INTAKE24 often (44\% compared with $15 \%$ who disagreed or strongly disagreed). Over $75 \%$ of participants agreed that INTAKE24 accurately captured their dietary intakes (Fig. 1) and over $80 \%$ agreed that the system was easy to follow and understand. A large majority of participants $(84 \%)$ disagreed that they would require help using INTAKE24 with only $3 \%$ stating that they would need help by either agreeing or strongly agreeing with this statement (Fig. 2).

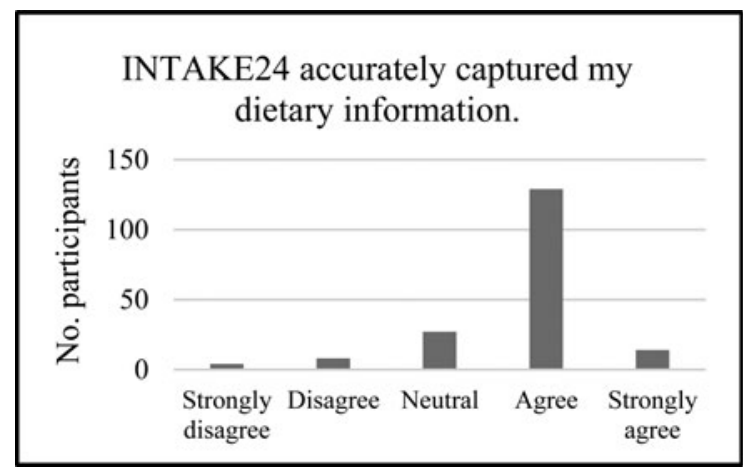

Fig. 1. Feedback on capturing of dietary data

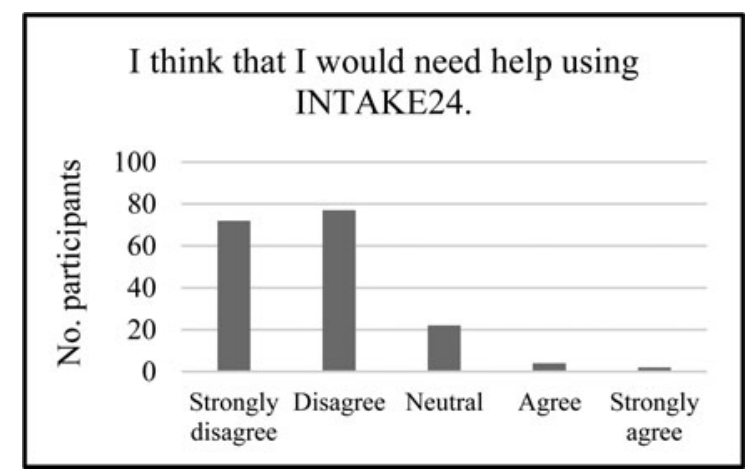

Fig. 2. Feedback on usability of the system

Feedback from participants was used to further enhance INTAKE24 ensuring suitability for collecting dietary intake from a diverse age range and population. A number of adaptations were made to the system: these included the addition of a video tutorial, context specific help, ability to input recipe items and missing foods (allowing the food database to be expanded) and the addition of a help page to answer users' commonly asked questions. The majority of participants $(75 \%)$ stated that they would like to receive dietary feedback and therefore this feature is currently being developed. The system is in a continuous state of being improved and is currently being adapted in a number of international contexts.

This work was funded by Food Standards Scotland.

1. Foster E, Delve J, Simpson E, Breininger S. Comparison study: INTAKE24 vs Interviewer led recall [Internet]. Food Standards Agency; 2014 [cited 2016 Mar 29] Available from: https://www.food.gov.uk/sites/default/files/INTAKE24\%20Comparison $\% 20$ study $\% 20$ final $\% 20 \mathrm{report}$.pdf

2. Food Standards Agency. Low Income Diet and Nutrition Survey, Volume 3. United Kingdom: The Stationary Office; 2007. 